\title{
ANÁLISE DE FREQÜÊNCIA HIDROLÓGICA DOS DADOS DE PRECIPITAÇÃO PLUVIAL DE ALGUMAS ESTAÇÕES AGROCLIMATOLÓGICAS DA REGIÃO SUL DO RIO GRANDE DO SUL
}

\author{
ANALYSIS OF HIDROLOGICAL FREQUENCY OF RAINFALL DATA OF SOME \\ AGROCLIMATOLOGICAL STATIONS IN SOUTH REGION OF RIO GRANDE DO SUL STATE
}

\author{
Rita de Cássia Fraga Damé ${ }^{1}$ Claudia Fernanda Almeida Teixeira ${ }^{2}$ Mery Vijagran Souto $^{2}$ \\ Núcia Margot Moreira²
}

\section{RESUMO}

\begin{abstract}
A análise de freqüência hidrológica, possibilita conhecer a magnitude de um evento para uma dada freqüencia de ocorrência, identificando se os dados observados ajustam-se a alguma distribuição de probabilidade. O presente trabalho teve como objetivos, ajustar os dados observados de precipitação pluvial anual e máximo e mínimo mensal, para diversos niveis de probabilidade, das estações agroclimatológicas de Pelotas, Morro Redondo, Domingos Petroline e Rio Grande. A série de valores de precipitação pluvial anual das estações agroclimatológicas de Pelotas, Morro Redondo, Domingos Petroline e Rio Grande, ajustaram-se adequadamente as distribuições de probabilidade normal, log-normal, log-normal e Gumbel, respectivamente. A série dos máximos anuais adequaram-se, para as estações descritas anteriormente, às distribuições de probabilidades de Gumbel, log-normal, Gumbel e log-normal, respectivamente. A série de minimos anuais de Rio Grande não foi considerada homogênea pelo "Run-Test".
\end{abstract}

Palavras-chave: chuva, distribuição, probabilidade.

\section{SUMMARY}

The hydrologic frequence analysis makes possible to know the magnitude of an event for a given ocorrency of frequence. It also identify wheather the observed data follow some theoretical distribuition of probability. The objective of this study was to adjust the observed data of annual rainfall, as well maximum and minimum monthly rainfall, in several probability levels, for the agroclimate stations of Pelotas, Morro Redondo, Domingos Petroline and Rio Grande. The series of annual rainfall data of Pelotas, Morro Redondo, Domingos Petroline and Rio Grande station were adjusted adequately to the normal, log-normal, lognormal and Gumbel distribuitions of probability, respectively. The series of annual maximum were adequate to the Gumbel, lognormal, Gumbel and log-normal distribuitions of probability, respectively. The series of annual minimum for Rio Grande station was not homogeneous by the "Run-Test".

Key words: rainfall, distribution, probability.

\section{INTRODUÇÃO}

O comportamento dos determinados elementos climáticos, como a precipitação, temperatura, vento, insolação e umidade relativa do ar, tem sido, há muito, objeto de estudo. A importância destes elementos, tem despertado grande interesse na interpretação, simulação e previsão dos mesmos. A precipitação pluvial, de modo geral e, especialmente, as precipitações pluviais máximas e mínimas apresentam grande interesse de ordem técnica por sua utilização em projetos de obras hidráulicas (BAPTISTA da SILVA \& AMARAL, 1987).

Uma das ferramentas que possibilita conhecer o comportamento da precipitação pluvial em uma dada freqüência de ocorrência é a análise de freqüência hidrológica, que consiste em analisar estatisticamente os dados coletados dos postos agroclimatológicos. Para que esta análise seja feita, é necessária a verificação da homogeneidade da série. De acordo com WMO (1966) e CLARKE (1993) os eventos hidrológicos seguem algum tipo de distribuição

\footnotetext{
${ }^{1}$ Engenheiro Agrícola, Mestre, Professor Assistente, Departamento de Engenharia Agrícola, Faculdade de Engenharia Agricola, Universidade Federal de Pelotas, (UFPel), Caixa Postal 354, 96010-900, Pelotas, RS. Autor para correspondência.

${ }^{2}$ Acadêmico, Departamento de Engenharia Agrícola, Faculdade de Engenharia Agrícola, UFPel.
} 
teórica de probabilidade assim, é possível demonstrar que, os dados observados no passado de uma dada variável hidrológica, seguem alguma distribuição teórica de probabilidade e as características conhecidas podem ser usadas para estimar a probabilidade de ocorrência de um evento para uma dada magnitude (GOULART, 1991; DAMÉ, 1995). Os mesmos autores afirmam que, deve ser observado que a distribuição teórica não é uma representação exata do processo natural, mas somente uma descrição aproximada do fenômeno.

As distribuições contínuas de probabilidades mais usadas em análise de freqüência hidrológica, de acordo com SANCHEZ (1987) são a distribuição Normal, log-normal, Gumbel e log-Pearson tipo III. Os objetivos deste trabalho foram ajustar os dados observados de precipitação pluvial a uma distribuição teórica de probabilidade e determinar os valores de precipitação pluvial anual e máxima e mínima mensal, para diversos níveis de probabilidade, de quatro estações agroclimatológicas da Região Sul do Rio Grande do Sul.

\section{MATERIAIS E MÉTODOS}

Utilizaram-se os dados de precipitação pluvial anual e máxima e mínima mensal das estações agroclimatológicas de Pelotas (Pelotas), Morro Redondo (Morro Redondo), Domingos Petroline (Rio Grande) e Rio Grande (Rio Grande). Para a estação de Rio Grande (Rio Grande) considerou-se somente os valores de precipitação pluvial anual e máxima mensal.

Os dados foram obtidos junto à Estação Agroclimatológica de Pelotas, convênio EMBRAPA/UFPel/INMET, e Agência de Desenvolvimento da Lagoa Mirim - Convênio ALM e Faculdade de Engenharia Agrícola - ambas da UFPel. Para a Estação de Pelotas a série de anos utilizada no trabalho foi de 1958 à 1990. Para Morro Redondo foi 1967 à 1987; Domingos Petroline 1956 à 1979 e Rio Grande 1939 à 1972.

A série dos valores de precipitação pluvial anual foi obtida através do somatório dos respectivos valores de cada ano de estudo. Já a série dos valores de precipitação pluvial máxima e mínima mensal obteve-se extraindo-se o maior e o menor valor mensal, respectivamente, da série completa de dados. Para verificar a homogeneidade das séries aplicou-se o "Run Test" (THOM, 1966).

As probabilidades empíricas acumuladas, foram determinadas pela fórmula de Weibull:
$\mathrm{P}(\mathrm{X} \geq \mathrm{X})=\mathrm{i} / \mathrm{N}+1$. Optou-se por este método ao invés do método da Califórnia por este último apresentar posição de plotagem igual a 1 , e uma vez que trabalhou-se com uma amostra de dados e não com a população, àquele é o mais indicado (GOULART, 1991).

A equação geral de análise de freqüência hidrológica de acordo com GOULART (1991) é dada pela seguinte expressão:

$$
P_{e}=\overline{P_{o}}+K_{t r} \cdot S_{o}
$$

onde $\mathrm{P}_{\mathrm{e}}$ é a precipitação pluvial em mm, estimada para um dado período de retorno; $\mathrm{P}_{\mathrm{o}}$ a média dos valores observados da precipitação pluvial em $\mathrm{mm} ; \mathrm{K}_{\mathrm{Tr}}$ o fator de freqüência tabelado para cada tipo de distribuição de probabilidade (GOULART, 1991; DAMÉ, 1995) e, $\mathrm{S}_{\mathrm{o}}$ é o desvio padrão dos valores observados da precipitação pluvial.

As distribuições teóricas de probabilidade aplicadas aos dados foram: distribuição normal, lognormal, Gumbel e log-Pearson tipo III. Para selecionar a distribuição mais adequada aos valores observados de precipitação pluvial, escolheu-se a distribuição que apresentou o menor erro padrão da estimativa $\left(S_{e}\right)$, apresentado por GOULART et al, 1992.

$$
S_{e}=\left[(1 / N) . \Sigma\left(P_{o}-P_{e}\right)^{2}\right]^{1 / 2}
$$

onde $\mathrm{P}_{\mathrm{o}}$ e $\mathrm{P}_{\mathrm{e}}$ são os valores observados e estimados de precipitação pluvial, para um certo período de retorno e $\mathrm{N}$ é o número de observações.

\section{RESULTADOS E DISCUSSÃO}

Para as estações agroclimatológicas de Pelotas (Pelotas), Morro Redondo (Morro Redondo), e Domingos Petroline (Rio Grande), as séries dos valores de precipitação pluvial anual e máxima e mínima mensal, mostraram-se homogêneas, pelo "Run Test". No entanto, a série dos valores de precipitação mínima mensal da estação agroclimatológica de Rio Grande (Rio Grande) foi heterogênea.

Através da análise de frequiência hidrológica,obtiveram-se os valores de precipitação pluvial estimados para um dado período de retorno, para as distribuições normal, log-normal, Gumbel e log-Pearson tipo III (Tabela 1), para as estações estudadas. 
Tabela 1. Equaçð̃es das distribuiçð̃es teóricas de probabilidade para os valores de precipitação anual máxima e mínima mensal das estaçð̃es agroclimatológicas consideradas.

\begin{tabular}{|c|c|c|c|c|}
\hline \multirow{2}{*}{$\begin{array}{l}\text { Estaçðes } \\
\text { agroclimatológicas }\end{array}$} & \multicolumn{4}{|c|}{ Distribuições teóricas s de probabilidade } \\
\hline & Normal & Log - Normal & Gumbel & Log-Pearson tipo III \\
\hline \multicolumn{5}{|l|}{ Anual } \\
\hline Pelotas & $P_{e}=1292,3+265,7 K_{r}$ & $P_{e}=3,1+0,09 \mathrm{KT}_{r}$ & $P_{e}=1292,3+265,7 \mathrm{KT}_{r}$ & $P_{e}=3,1+0,09 \mathrm{KT}_{\mathrm{r}}$ \\
\hline M. Redondo & $P_{e}=1531,7+215,3 \mathrm{KT}_{r}$ & $P_{e}=3,2+0,06 \mathrm{KT}_{\mathrm{r}}$ & $P_{e}=1531,7+215,3 \mathrm{KT}_{\mathrm{r}}$ & $\mathrm{P}_{\mathrm{e}}=3,2+0,06 \mathrm{KT}_{\mathrm{r}}$ \\
\hline D. Petroline & $P_{e}=1353,8+260,0 \mathrm{KT}_{r}$ & $P_{e}=3,1+0,08 \mathrm{KT}_{r}$ & $P_{e}=1353,8+260,0 \mathrm{KT}_{\mathrm{r}}$ & $\mathrm{P}_{\mathrm{e}}=3,1+0,08 \mathrm{KT}_{\mathrm{r}}$ \\
\hline Rio Grande & $P_{e}=1243,9+276,8 \mathrm{KT}_{r}$ & $\mathbf{P}_{\mathrm{e}}=3,1+0,09 \mathrm{KT}$ & $P_{e}=1243,9+276,8 \mathrm{KT}_{r}$ & $P_{e}=3,1+0,09 \mathrm{KT}_{\mathrm{r}}$ \\
\hline \multicolumn{5}{|l|}{ Máximo mensal } \\
\hline Pelotas & $P_{e}=241,7+77,4 \mathrm{KT}_{r}$ & $P_{e}=2,4+0,13 \mathrm{KT}_{r}$ & $\mathrm{P}_{\mathrm{e}}=241,7+77,4 \mathrm{KT}_{\mathrm{r}}$ & $P_{e}=2,4+0,13 K T_{r}$ \\
\hline M. Redondo & $\mathrm{Pe}=266,0+62,2 \mathrm{KT}_{\mathrm{r}}$ & $P_{e}=2,4+0,10 \mathrm{KT}_{\mathrm{r}}$ & $P_{e}=266,0+62,2 \mathrm{KT}_{r}$ & $P_{e}=2,4+0,10 \mathrm{KT}_{r}$ \\
\hline D. petroline & $\mathrm{Pe}=235,3+77,1 \mathrm{KT}_{\mathrm{r}}$ & $P_{e}=2,4+0,12 \mathrm{Ktr}$ & $P_{e}=235,3+77,1 \mathrm{Ktr}$ & $\mathrm{P}_{\mathrm{e}}=2,3+0,12 \mathrm{KT}_{\mathrm{r}}$ \\
\hline Rio Grande & $\mathrm{Pe}=222,3+60,5 \mathrm{KTr}$ & $\mathrm{P}_{\mathrm{e}}=2,3+0,12 \mathrm{KT}_{\mathrm{r}}$ & $\mathrm{P}_{\mathrm{e}}=222,3+60,5 \mathrm{KT}_{\mathrm{r}}$ & $\mathrm{P}_{\mathrm{e}}=2,3+0,12 \mathrm{KT}_{\mathrm{r}}$ \\
\hline \multicolumn{5}{|l|}{ Mínimo mensal } \\
\hline Pelotas & $P_{e}=24,7+12,35 \mathrm{KT}_{r}$ & $\mathrm{P}_{\mathrm{e}}=1,3+0,24 \mathrm{KT}_{\mathrm{r}}$ & $P_{e}=24,7+12,3 \mathrm{KT}_{\mathrm{r}}$ & $\mathrm{P}_{\mathrm{e}}=1,3+0,24 \mathrm{KT} \mathrm{r}_{\mathrm{r}}$ \\
\hline M. Redondo & $\mathrm{Pe}=35,5+20,7 \mathrm{KT}_{\mathrm{r}}$ & $P_{e}=1,5+0,33 \mathrm{KT}_{r}$ & $\mathrm{P}_{\mathrm{e}}=35,5+20,7 \mathrm{KT}_{\mathrm{r}}$ & $\mathrm{P}_{\mathrm{e}}=1,5+0,33 \mathrm{KT}_{\mathrm{r}}$ \\
\hline D. Petroline & $\mathrm{Pe}=27,7+14,7 \mathrm{KTr}$ & $P e=1,4+0,19 \mathrm{KT}_{\mathrm{r}}$ & $P_{e}=27,7+14,7 K_{r}$ & $\mathrm{P}_{\mathrm{e}}=1,4+0,19 \mathrm{KT}_{\mathrm{r}}$ \\
\hline
\end{tabular}

Para os valores de precipitação pluvial anual das estações agroclimatológicas de Pelotas, Morro Redondo e Domingos Petroline as distribuições que melhor ajustaram-se aos dados foi a normal e lognormal (Tabela 2). LANNA (1993) afirma que os valores de precipitação anual, costumam ajustar-se adequadamente à distribuição normal, enquanto que GOULART (1991) explica que muitas vezes a variável não se ajusta a distribuição normal, mas o logarítmo desta, ajusta-se. Desta forma, os resultados encontrados concordam com o descrito em LANNA (1993) e GOULART (1991). Já para a estação de Rio Grande, a distribuição de Gumbel foi a que apresentou o menor erro padrão da estimativa. Esta distribuição caracteriza-se como distribuição de eventos extremos (SANCHEZ, 1987; GOULART, 1991; LANNA, 1993), logo não esperava-se que para esta série a distribuição de Gumbel, fosse a mais adequada. Embora não tenha sido feito nenhum teste estatístico para verificar se há diferença significativa entre os valores do erro padrão da estimativa encontrados para as distribuições normal, log-normal e Gumbel, estes são muito próximos, conforme pode ser observado na Tabela 2. Os valores de precipitação pluvial máxima mensal para as estações agroclimatológicas de Pelotas e Domingos Petroline, ajustaram-se mais adequadamente à distribuição de Gumbel, conforme o esperado e de acordo com CLARKE (1993) eLANNA (1993).
Para os valores de precipitação pluvial mínima mensal, a distribuição de Gumbel ajustou-se melhor nas estações agroclimatológicas de Morro Redondo, Domingos Petroline e Rio Grande.

Tabela 2. Valores do erro padrão da estimativa (Se) para dados de precipitacão anual e máxima e mínima mensal, para as distribuições Normal, Log-Normal, Gumbel e Log-Pearson tipo III, observados nas estaç̃̃es agroclimatológicas consideradas.

\begin{tabular}{|c|c|c|c|c|}
\hline \multirow[b]{2}{*}{$\begin{array}{l}\text { Estaçס̃es } \\
\text { Agroclima- } \\
\text { tológicas }\end{array}$} & \multicolumn{3}{|c|}{ Distribuiçð̃es de probabilidade } & \multirow[b]{2}{*}{$\begin{array}{l}\text { Log-Pearson } \\
\text { tipo III }\end{array}$} \\
\hline & Normal & Log-Normal & Gumbel I & \\
\hline \multicolumn{5}{|l|}{ Anual } \\
\hline $\begin{array}{l}\text { Pelotas } \\
\text { M. Redondo } \\
\text { D. Petroline } \\
\text { Rio Grande }\end{array}$ & $\begin{array}{l}2,4773^{*} \\
55,5870 \\
50,2820 \\
58,1250\end{array}$ & $\begin{array}{l}18,6356 \\
55,2826^{*} \\
40,9240^{*} \\
43,9910\end{array}$ & $\begin{array}{l}21,9923 \\
64,4982 \\
42,1080 \\
40,9430^{*}\end{array}$ & $\begin{array}{l}93,2024 \\
79,5817 \\
66,9897 \\
78,7015\end{array}$ \\
\hline \multicolumn{5}{|c|}{ Máxima Mensal } \\
\hline $\begin{array}{l}\text { Pelotas } \\
\text { M. Redondo } \\
\text { D. Petroline } \\
\text { Rio Grande }\end{array}$ & $\begin{array}{l}14,4865 \\
18,4759 \\
35,9350 \\
14,0096\end{array}$ & $\begin{array}{l}12,1101 \\
15,5326^{*} \\
31,8750 \\
11,6111^{*}\end{array}$ & $\begin{array}{l}8,8002^{*} \\
15,7270 \\
27,4170^{*} \\
12,5149\end{array}$ & $\begin{array}{l}29,2114 \\
22,0846 \\
34,2252 \\
20,1523\end{array}$ \\
\hline \multicolumn{5}{|c|}{ Mínima Mensal } \\
\hline $\begin{array}{l}\text { Pelotas } \\
\text { M. Redondo } \\
\text { D. Petroline }\end{array}$ & $\begin{array}{l}0,2662^{*} \\
4,5568 \\
7,6807\end{array}$ & $\begin{array}{l}0,6978 \\
6,3139 \\
6,0915\end{array}$ & $\begin{array}{l}0,6405 \\
3,8770^{*} \\
5,4736^{*}\end{array}$ & $\begin{array}{l}4,5166 \\
7,3423 \\
5,9319\end{array}$ \\
\hline
\end{tabular}

* Menor valor do erro padrão da estimativa. 
Nas Tabelas 3, 4, 5 e 6 encontram-se os valores estimados de precipitação pluvial anual e máxima e mínima mensal $(\mathrm{mm})$ das estações agroclimatológicas em estudo. Estas tabelas apresentam, a freqüência, com que ocorrem valores iguais ou superiores aos valores de uma série temporal. Assim, por exemplo, tem-se uma garantia de $97 \%$ de que os valores de precipitação anual de Pelotas assumem um valor não menos que $784,5 \mathrm{~mm}$, os valores de precipitação máxima mensal de Morro Redondo, assumem um valor não menos que $181,1 \mathrm{~mm}$ isto com $95 \%$ de certeza, e assim sucessivamente.

Tabela 3. Valores estimados de precipitação pluvial anual e máxima e mínima mensal $(\mathrm{mm})$ em função dos níveis de probabilidade da estação agroclimatológica de Pelotas, obtidos através da distribuição teórica de probabilidade Normal, Gumbel e Normal, respectivamente.

\begin{tabular}{|c|c|c|c|}
\hline$P(X \geq X)=i / N+1$ & Anual & $\begin{array}{l}\text { Máxima } \\
\text { Mensal }\end{array}$ & $\begin{array}{l}\text { Mínima } \\
\text { Mensal }\end{array}$ \\
\hline
\end{tabular}

\begin{tabular}{|c|c|c|c|}
\hline 0,97 & 784,5 & 130,6 & 5,3 \\
\hline 0,94 & 810,5 & 143,2 & 6,8 \\
\hline 0,91 & 845,0 & 154,0 & 9,4 \\
\hline 0,88 & 964,6 & 160,3 & 10,4 \\
\hline 0,85 & 991,4 & 167,2 & 10,8 \\
\hline 0,82 & 1025,9 & 173,0 & 11,2 \\
\hline 0,79 & 1047,5 & 179,3 & 11,7 \\
\hline 0,76 & 1114,8 & 184,8 & 12,3 \\
\hline 0,74 & 1135,3 & 189,7 & 12,9 \\
\hline 0,71 & 1159,4 & 194,9 & 13,6 \\
\hline 0,68 & 1182,1 & 199,7 & 14,4 \\
\hline 0,65 & 1198,8 & 204,7 & 15,3 \\
\hline 0,62 & 1211,4 & 209,3 & 16,4 \\
\hline 0,59 & 1225,9 & 214,1 & 17,6 \\
\hline 0,56 & 1257,1 & 219,0 & 19,1 \\
\hline 0,53 & 1276,2 & 224,0 & 21,5 \\
\hline 0,50 & 1295,1 & 229,0 & 23,2 \\
\hline 0,47 & 1317,5 & 234,4 & 23,4 \\
\hline 0,44 & 1337,8 & 239,7 & 23,7 \\
\hline 0,41 & 1356,1 & 245,2 & 24,0 \\
\hline 0,38 & 1375,3 & 251,1 & 24,3 \\
\hline 0,35 & 1394,2 & 257,0 & 24,7 \\
\hline 0,32 & 1418,5 & 263,5 & 25,1 \\
\hline 0,29 & 1444,2 & 270,5 & 25,7 \\
\hline 0,26 & 1466,7 & 278,1 & 26,4 \\
\hline 0,24 & 1491,0 & 286,3 & 27,4 \\
\hline 0,21 & 1518,9 & 295,5 & 28,6 \\
\hline 0,18 & 1553,1 & 305,8 & 30,3 \\
\hline 0,15 & 1580,5 & 317,8 & 33,0 \\
\hline 0,12 & 1615,2 & 332,3 & 37,2 \\
\hline 0,09 & 1659,0 & 350,6 & 41,9 \\
\hline 0,06 & 1715,4 & 376,1 & 43,5 \\
\hline 0,03 & 1805,7 & 418,8 & 48,3 \\
\hline
\end{tabular}

Tabela 4. Valores estimados de precipitação pluvial anual e máxima e mínima mensal(mm), em função dos níveis de probabilidade da estação agroclimatológica de Morro Redondo (Morro Redondo), obtidos através da distribuição teórica de probabilidade LogNormal, Log-Normal e Gumbel, respectivamente.

\begin{tabular}{cccc}
\hline $\mathrm{P}(\mathrm{X} \geq \mathrm{X})=\mathrm{i} / \mathrm{N}+1$ & Anual & $\begin{array}{c}\text { Máxima } \\
\text { Mensal }\end{array}$ & $\begin{array}{c}\text { Mínioma } \\
\text { Mensal }\end{array}$ \\
\hline 0,95 & 1205,3 & 181,1 & 8,2 \\
0,91 & 1266,6 & 195,8 & 12,1 \\
0,86 & 1296,2 & 203,0 & 15,2 \\
0,82 & 1331,3 & 211,7 & 17,5 \\
0,77 & 1376,1 & 222,9 & 19,7 \\
0,73 & 1401,9 & 229,5 & 22,1 \\
0,68 & 1427,9 & 236,2 & 24,1 \\
0,64 & 1446,7 & 241,1 & 25,9 \\
0,59 & 1463,1 & 245,3 & 28,0 \\
0,55 & 1494,9 & 253,8 & 30,0 \\
0,50 & 1517,3 & 259,7 & 32,1 \\
0,45 & 1544,4 & 267,0 & 34,3 \\
0,41 & 1567,1 & 273,2 & 36,5 \\
0,36 & 1590,7 & 279,6 & 39,0 \\
0,32 & 1620,9 & 288,0 & 41,7 \\
0,27 & 1651,6 & 296,6 & 44,7 \\
0,23 & 1684,2 & 305,7 & 48,1 \\
0,18 & 1722,9 & 316,8 & 52,1 \\
0,14 & 1767,3 & 329,7 & 57,2 \\
0,09 & 1826,8 & 347,2 & 64,1 \\
0,05 & 1921,3 & 375,7 & 75,7 \\
\hline
\end{tabular}

Tabela 5. Valores estimados da precipitação pluvial anual e máxima e mínima mensal (mm), em função dos níveis de probabilidade da estação agroclimatológica de Domingos Petroline (Rio grande), obtidos através da distribuição teórica de probabilidade LogNormal, Gumbel e Gumbel, respectivamente.

\begin{tabular}{cccc}
\hline $\mathrm{P}(\mathrm{X} \geq \mathrm{X})=\mathrm{i} / \mathrm{N}+1$ & Anual & $\begin{array}{c}\text { Máxima } \\
\text { Mensal }\end{array}$ & $\begin{array}{c}\text { Minima } \\
\text { Mensal }\end{array}$ \\
\hline 0,96 & 969,1 & 130,4 & 7,6 \\
0,92 & 1025,3 & 144,7 & 10,7 \\
0,88 & 1064,2 & 155,3 & 12,6 \\
0,84 & 1095,5 & 164,2 & 14,2 \\
0,80 & 1135,6 & 172,0 & 15,7 \\
0,76 & 1176,6 & 179,2 & 17,1 \\
0,72 & 1201,3 & 186,1 & 18,4 \\
0,68 & 1227,3 & 192,7 & 19,6 \\
0,64 & 1246,7 & 199,3 & 20,8 \\
0,60 & 1263,1 & 205,8 & 22,1 \\
0,56 & 1294,8 & 212,5 & 23,4 \\
0,52 & 1318,6 & 219,2 & 24,6 \\
0,48 & 1343,6 & 226,1 & 26,0 \\
0,44 & 1370,7 & 233,4 & 27,3 \\
0,40 & 1395,9 & 241,0 & 28,8 \\
0,36 & 1420,4 & 249,1 & 30,3 \\
0,32 & 1452,5 & 257,9 & 32,0 \\
0,28 & 1485,5 & 267,5 & 33,8 \\
0,24 & 1519,7 & 278,3 & 35,9 \\
0,20 & 1560,5 & 290,7 & 38,3 \\
0,16 & 1606,0 & 305,6 & 41,1 \\
0,12 & 1656,7 & 324,3 & 44,6 \\
0,08 & 1725,5 & 350,0 & 49,5 \\
0,04 & 1844,0 & 392,2 & 57,7 \\
& & & \\
\hline
\end{tabular}


Tabela 6. Valores estimados de precipitação pluvial anual e máxima mensal $(\mathrm{mm})$, em função dos níveis de probabilidade da estação agroclimatológica de Rio Grande (Rio grande), obtidos através da distribuição teórica de probabilidade Gumbel e Log-Normal, respectivamente.

\begin{tabular}{lll}
\hline $\mathrm{P}(\mathrm{X} \geq \mathrm{X})=\mathrm{i} / \mathrm{N}+1$ & Anual & Máxima Mensal \\
\hline & & \\
\hline 0,97 & 843,9 & 132,2 \\
0,94 & 891,5 & 140,2 \\
0,91 & 924,7 & 149,2 \\
0,89 & 951,4 & 154,3 \\
0,86 & 974,8 & 158,9 \\
0,83 & 996,1 & 164,1 \\
0,80 & 1015,8 & 170,5 \\
0,77 & 1034,5 & 177,7 \\
0,74 & 1052,4 & 181,7 \\
0,71 & 1069,9 & 185,8 \\
0,69 & 1086,9 & 189,9 \\
0,66 & 1103,9 & 193,8 \\
0,63 & 1120,6 & 196,2 \\
0,60 & 1137,4 & 198,9 \\
0,57 & 1154,3 & 203,6 \\
0,54 & 1171,4 & 208,7 \\
0,51 & 1188,7 & 212,4 \\
0,49 & 1206,6 & 216,7 \\
0,46 & 1224,9 & 221,5 \\
0,43 & 1243,8 & 225,5 \\
0,40 & 1263,5 & 229,9 \\
0,37 & 1284,1 & 233,9 \\
0,34 & 1305,8 & 238,8 \\
0,31 & 1328,9 & 245,9 \\
0,29 & 1353,6 & 250,6 \\
0,26 & 1380,4 & 256,3 \\
0,23 & 1409,7 & 262,5 \\
0,20 & 1442,3 & 270,2 \\
0,17 & 1479,2 & 277,8 \\
0,14 & 1522,1 & 286,7 \\
0,11 & 1573,7 & 297,1 \\
0,09 & 1639,2 & 309,9 \\
0,06 & 1729,9 & 328,5 \\
0,03 & 1882,8 & 359,3 \\
& & \\
\hline & & \\
\hline
\end{tabular}

\section{CONCLUSÕES}

As distribuições de probabilidade que melhor se ajustam aos dados de precipitação anual da estação agroclimatológica de Pelotas (Pelotas) é a Normal, e para as estações agroclimatológicas de Morro Redondo (Morro Redondo), Domingos Petroline (Rio Grande) e Rio Grande (Rio Grande) é a Log-Normal.

As distribuições de probabilidade que melhor se ajustam aos dados de precipitação máxima mensal das estações agroclimatológicas Log-Normal, e para as estações agroclimatológicas de Pelotas (Pelotas), e Domingos Petroline (Rio Grande) é a Gumbel.

A distribuição de probabilidade que melhor se ajusta aos dados de precipitação mínima mensal da estação agroclimatológica de Pelotas (Pelotas) é a Normal, e para as estações agroclimatológicas de Morro Redondo (Morro Redondo) e Domingos Petroline (Rio Grande) e Rio Grande (Rio Grande) é a Gumbel.

\section{REFERÊNCIAS BIBLIOGRÁFICAS}

BAPTISTA da SILVA, J., AMARAL, E. Probabilidades das precipitações pluviométricas em Pelotas, RS. Revista Brasileira de Meteorologia, São Paulo, v. 2, p. 167-177. 1987.

CLARKE, R.T. Hidrologia - Ciência e aplicação. Porto Alegre Universidade Federal do Rio Grande do Sul, 1993. v. 4 hidrologia estatística: p. $659-698$.

DAMÉ, R.C.F. Apostila de hidrologia. Pelotas: UFPel, FEA, 1995. $134 \mathrm{p}$

GOUlart, J.P. Apostila de hidrologia. Pelotas: UFPel, FEA, 1991. $194 \mathrm{p}$

GOULART, J.P., MAESTRINI, A.P., NEBEL, A. L. Relação intensidade-duração-freqüência de chuvas em Pelotas, RS Revista Brasileira de Meteorologia, São Paulo. v. 7, n. 1, p 543-552. 1992.

LANNA, A.E. Hidrologia - Ciência e aplicação. Porto Alegre Universidade Federal do Rio Grande do Sul, 1993. v.4 elementos de estatística e probabilidades: p. 79-164

LINSLEY, R.K., FRANZINI, J.B. Engenharia de recursos hídricos. São Paulo: Mcgraw-Hill do Brasil, 1978. 786 p.

SANCHEZ, J. Fundamentos de hidrologia. Porto Alegre Institutos de Pesquisas Hidráulicas, UFRGS, 1987. 360 p

WMO Word Meteorological Organization. Some methods of climatological analysis. Genebra: World Meteorological analysis, 1966. 53 p. (Technical Note ${ }^{\circ} 81$ ).

Ciência Rural, v. 26, n. 3, 1996. 